\title{
Association Between Chronologic Age and Geriatric Assessment-Identified Impairments: Findings From the CARE Registry
}

\author{
Smith Giri, MD, MHS ${ }^{1,2,3}$; Mustafa Al-Obaidi, MBBS ${ }^{1}$; Alice Weaver, MD³; Kelly M. Kenzik, PhD 1,2,3; \\ Andrew McDonald, MD, $\mathrm{MS}^{1,2}$; Deanna Clark, MBA, MPH${ }^{1}$; Crystal Young-Smith, CRNP ${ }^{3}$; \\ Ravi Paluri, MD, $\mathrm{MPH}^{2,3}$; Lakshmin Nandagopal, $\mathrm{MD}^{2,3}$; Olumide Gbolahan, MD², ${ }^{2,3}$ Mackenzi Pergolotti, $\mathrm{PhD}^{4,5}$; \\ Smita Bhatia, MD, MPH ${ }^{1,2}$; and Grant R. Williams, $\mathrm{MD}^{1,2,3}$
}

\begin{abstract}
Background: The NCCN Guidelines for Older Adult Oncology recommend that, when possible, older adults with cancer undergo a geriatric assessment (GA) to provide a comprehensive health appraisal to guide interventions and appropriate treatment selection. However, the association of age with GA-identified impairments (GA impairments) remains understudied and the appropriate age cutoff for using the GA remains unknown. Patients and Methods: We designed a cross-sectional study using the Cancer and Aging Resilience Evaluation (CARE) registry of older adults with cancer. We included adults aged $\geq 60$ years diagnosed with gastrointestinal malignancy who underwent a patient-reported GA prior to their initial consultation at the gastrointestinal oncology clinic. We noted the presence of GA impairments and frailty using Rockwood's deficit accumulation approach. We studied the relation between chronologic age and GA impairments/ frailty using Spearman rank correlation and chi-square tests of trend. Results: We identified 455 eligible older adults aged $\geq 60$ years with gastrointestinal malignancies; the median age was 68 years (range, 64-74 years) and colorectal (33\%) and pancreatic (24\%) cancers were the most common cancer type. The correlation between chronologic age and number of geriatric impairments was weak and did not reach statistical significance (Spearman $\rho, 0.07 ; P=.16$ ). Furthermore, the prevalence of domain-specific impairments or frailty was comparable across the 3 age groups (60-64 years, 65-74 years, $\geq 75$ years) with the exception of comorbidity burden. Notably, $61 \%$ of patients aged 60 to 64 years had $\geq 2 \mathrm{GA}$ impairments and $35 \%$ had evidence of frailty, which was comparable to patients aged 65 to 74 years ( $66 \%$ and $36 \%$, respectively) and $\geq 75$ years (70\% and $40 \%$, respectively). Conclusions: Using chronologic age alone to identify which patients may benefit from GA is problematic. Future studies should identify screening tools that may identify patients at high risk of frailty and GA impairments.
\end{abstract}

J Natl Compr Canc Netw 2021;19(8):922-927 doi: $10.6004 /$ jnccn.2020.7679

${ }^{1}$ Institute for Cancer Outcomes and Survivorship, ${ }^{2} \mathrm{O}^{\prime} \mathrm{Neal}$ Comprehensive Cancer Center, and ${ }^{3}$ Division of Hematology and Oncology, Department of Medicine, University of Alabama at Birmingham, Birmingham, Alabama; ${ }^{4}$ Revital Cancer Rehabilitation, Select Medical, Mechanicsburg, Pennsylvania; and ${ }^{5}$ Department of Occupational Therapy, Colorado State University, Fort Collins, Colorado.

\section{Background}

Up to $60 \%$ of all new cancer diagnoses and $70 \%$ of all cancer-related deaths occur among adults aged $\geq 65$ years. ${ }^{1}$ Older adults with cancer are at high risk of treatment-related toxicity and inferior survival, yet neither chronologic age nor clinician-assessed performance status (PS) adequately captures this vulnerability. ${ }^{2}$ A geriatric assessment (GA) is a multidimensional tool to uncover this vulnerability or frailty and predicts the risk of morbidity and mortality among older adults with cancer. ${ }^{3-5}$ A growing body of literature shows that GA can predict chemotherapy-related toxicity, ${ }^{5,6}$ as well as mortality, ${ }^{4}$ guide clinical decisionmaking, ${ }^{7}$ and improve patient satisfaction ${ }^{8}$ among older adults with cancer. Specifically among gastrointestinal cancers, a GA has been shown to predict postsurgical complications among older adults with colorectal cancer ${ }^{9}$ and gastroesophageal ${ }^{10}$ and hepatocellular carcinoma, ${ }^{11}$ as well as chemotherapy-related toxicity ${ }^{12}$ and short- and long-term mortality. ${ }^{13-15}$ Given these substantial benefits, $\mathrm{ASCO}^{16}$ and NCCN guidelines ${ }^{17}$ currently recommend that all older adults with cancer undergo a GA.

However, the association between chronologic age and GA impairments and frailty remains understudied. Furthermore, ASCO recommends a GA should be performed among all older adults with cancer aged $>65$ years, yet the rationale for this age cutoff remains unclear. Because patients with cancer are known to undergo accelerated aging through multiple mechanisms, ${ }^{18}$ extrapolating from the age cutoff used in the general population may be inaccurate. In this study, we examined the association between chronologic age and GA-identified impairments and frailty among adults aged $\geq 60$ years with gastrointestinal malignancies.

\section{See JNCCN.org for supplemental online content.}




\section{Patients and Methods}

\section{Study Population}

Using participants from the University of Alabama at Birmingham (UAB) Cancer and Aging Resilience Evaluation (CARE) Study, an ongoing prospective registry enrolling older adults ( $\geq 60$ years old) undergoing cancer care at UAB Hospitals and Clinics, ${ }^{19,20}$ we identified patients diagnosed with a gastrointestinal malignancy presenting to our medical oncology clinic for an initial consultation. We chose 60 years of age as a criterion for enrollment in this registry given the uncertainty surrounding the "right" age cutoff and to allow for meaningful age-related subanalyses, such as the current study. ${ }^{21}$ The UAB Institutional Review Board (IRB-300000092) approved this study.

\section{Geriatric Assessment}

We conducted patient-reported GAs as previously described (supplemental eTable 1; available with this article at JNCCN.org). ${ }^{19,20}$ Our GA comprised the following domains: functional status, comorbidity, cognition, mental health status, nutrition, social support, and health-related quality of life, consistent with recommendations from the International Society of Geriatric Oncology (SIOG). ${ }^{3}$ We assessed functional status using the Older Americans Resources and Services (OARS) instrumental activities of daily living (IADLs), ${ }^{22}$ OARS activities of daily living (ADLs), ${ }^{22}$ patient-reported ECOG PS, ${ }^{23}$ and number of falls within the last 6 months. ${ }^{24}$ Nutritional status was evaluated using an abridged version of patient-generated subjective global assessment (PG-SGA) ${ }^{25}$ Comorbidity assessment was performed using number of medications ${ }^{26}$ and OARS comorbidity assessment. ${ }^{22,27}$ We assessed social support using Medical Outcomes Study-Social Support Survey, ${ }^{28}$ mental health status using the PatientReported Outcomes Measurement Information System (PROMIS) Anxiety and Depression measures, ${ }^{29,30}$ and cognition using the PROMIS Cognitive Function measure. ${ }^{31}$ Lastly, health-related quality of life was examined using the PROMIS 10-item Global Health tool. ${ }^{32}$ The GA was completed by the patient. However, if the patient had any vision issues, a member of the study personnel or a primary caregiver could ask the patient the GA questions.

\section{GA Impairments}

Based on the GA evaluation, patients were classified as having GA impairment if they met $\geq 2$ of the following criteria $^{33}: \geq 1$ falls in the last 6 months, significant limitation in walking 1 block, impairment in $\geq 2$ IADLs, any ADL impairment, significant weight loss (3\% in 3 months or $6 \%$ within 6 months), presence of $\geq 4$ comorbidities, poor social support for physical activity, significant interference in social activity, presence of anxiety ( $T$-score $\geq 60$ ) or depression ( $\mathrm{T}$-score $\geq 60$ ), cognitive impairment (T-score $\leq 60$ ), or polypharmacy ( $\geq 9$ medications).

\section{Frailty Index}

We constructed a frailty index (the CARE Frailty Index) using the principle of deficit accumulation approach originally described by Rockwood and Mitnitski ${ }^{34}$ and following the standard procedures outlined by Searle et al. ${ }^{35}$ Similar methods have been used by Guerard et $\mathrm{al}^{4}$ and Cohen et $\mathrm{al}^{36}$ to construct frailty indices that have been shown to be predictive of chemotherapy toxicity ${ }^{36}$ and all-cause mortality ${ }^{4}$ among older adults with cancer. We selected 44 GA variables from the CARE questionnaire, each of which captured a health deficit, and recoded responses using the convention that 0 indicated the absence of the deficit and 1 indicated the presence of deficit; for variables that included a single intermediate response (eg, "sometimes" or "maybe"), we used an additional value of 0.5 . We then combined these individual scores into an aggregate frailty score reflecting the overall proportion of deficits (range, $0-1$ ), where $0=$ no deficit present and $1=$ all 44 deficits present. We then categorized patients as robust $(<0.2)$, prefrail $(0.2-0.35)$, and frail $(>0.35)$, as previously described. ${ }^{35}$ In case of missing response data, we required that responses to at least 30 items be present to construct a valid frailty index. An index constructed with at least 30 variables has been previously shown to be sufficiently accurate for predicting adverse outcomes among older adults. ${ }^{37}$ Additional details regarding our definition for GA impairment and construction of the CARE Frailty Index are provided in supplemental eAppendices 1 and 2 .

\section{Statistical Analysis}

We compared baseline characteristics between the 3 age groups (60-64, 65-74, and $\geq 75$ years) using appropriate bivariate statistical tests, namely, analysis of variance/ Kruskal Wallis for continuous variables and chi-square test/Fisher exact test for categorical variables depending on their underlying distribution. To measure the correlation between the number of geriatric impairments (a ranked variable) and chronologic age (continuous variable), we used Spearman rank correlation coefficient and tested the alternative hypothesis that the Spearman $\rho$ was significantly different from 0 . We compared the difference in proportion of various GA impairments and frailty categories among increasing age groups (60-64, $65-74$, and $\geq 75$ years) using chi-square tests of trend. To evaluate the difference between number of GA impairments across the 3 age groups, we used a nonparametric extension of Wilcoxon rank-sum test. ${ }^{38}$ All statistical tests were 2-sided and the level of significance was 0.05 . We used STATA, version 13 (StataCorp LLC) for all statistical analysis. 


\section{Results}

Of the 523 consecutive adults aged $\geq 60$ years with gastrointestinal malignancy seen for initial consultation at the UAB medical oncology clinic between September 2017 and October 2019, 455 (87\%) enrolled in the CARE registry and underwent GA (supplemental eFigure 1). Of these, 367 (81\%) had not started any systemic therapy, whereas the remaining (19\%) had previously received treatment elsewhere. The median age of the entire cohort at the time of GA was 68 years (interquartile range [IQR], $64-74$ years); $55 \%$ were male and $72 \%$ were nonHispanic White. Overall, $28 \%$ of the 455 patients were aged $60-64$ years, $47 \%$ were aged $65-74$ years, and $25 \%$ were aged $\geq 75$ years. Common cancer types included colorectal (33\%) and pancreatic (24\%); $46 \%$ had stage IV disease. The demographic and clinical characteristics were similar across the 3 age groups, with the exception of marital status and cancer stage, as summarized in Table 1. Compared with nonparticipants, patients enrolled in the CARE registry had similar age, sex, and cancer stage, with the exception of a higher proportion of nonresponders among patients with hepatobiliary and pancreatic cancer (supplemental eTable 2).

\section{Relationship Between Chronologic Age and \\ Geriatric Impairments}

There was no significant correlation between chronologic age and number of geriatric impairments (Spearman $\rho$, $0.07 ; P=.16$ ). Notably, even in the age group of $60-64$ years, $61.4 \%$ of patients had GA impairments. This was not significantly different compared with patients aged $65-74$ years $(66.2 \%)$ and $\geq 75$ years $(70.5 \% ; P=.11)$. We found similar rates of impairments in IADLs, ADLs, nutritional status, falls, cognitive, anxiety, depression, polypharmacy, and patient-reported ECOG PS across the age groups. However, there was a higher comorbidity burden $(\geq 3)$ in the older group $(39 \%, 56 \%$, and $56 \%$ among ages 60-64 years, $65-74$ years, and $\geq 75$ years, respectively; $P<.01$ ) (Table 2). The increased comorbidity burden in the older age groups was mainly driven by a higher proportion of patients reporting arthritis, hypertension, and glaucoma (supplemental eTable 3).

\section{Relationship Between Chronologic Age and Frailty} Overall, $37 \%(n=108)$ of the cohort were frail, whereas $30 \%(n=128)$ were prefrail and $33 \%(n=143)$ had robust frailty status. Compared with those who were prefrail or robust, patients who were frail were more likely to have an ECOG PS $\geq 2$ ( $68 \%$ vs $26 \%$ vs $4 \%$, respectively; $P<.001$ ) and a higher cancer stage ( $52 \%$ vs $42 \%$ vs $42 \%$, respectively; $P=.03$ ), but did not differ significantly by treatment status ( $22 \%$ vs $20 \%$ vs $16 \%$, respectively, were already on treatment; $P=.44$ ).
We then compared the rates of frailty categories across the different age groups. Notably, $26 \%$ and $35 \%$ of patients aged 60-64 years had evidence of prefrail and frail status. This was not significantly different compared with the proportion of patients with prefrail and frail status in the $65-74$ age group $(30 \%$ and $36 \%$, respectively) and $\geq 75$ age group (33\% and $40 \%$, respectively) $(P=.45)$.

\section{Discussion}

In this study comprising an unselected cohort of older adults aged $\geq 60$ years with gastrointestinal malignancies, we found no significant relationship between chronologic age and the presence of geriatric impairments or frailty. Furthermore, we found comparable prevalence of GA impairments and frailty in the 60-64 age group compared with those aged $\geq 65$ years, suggesting that the traditional cutoff of 65 years for conducting comprehensive GA may not be accurate, and even patients aged $<65$ years could benefit from GA evaluation.

There is no universal agreement on the age at which a person becomes old. In the United States, age $\geq 65$ years is generally considered the chronologic definition of an older adult, similar to what is used for Medicare eligibility. Accordingly, consensus recommendations for GA from ASCO and SIOG use 65 years as the age cutoff., ${ }^{3,16,17}$ However, emerging evidence suggests that cancer diagnosis and/or treatment can accelerate the human aging process through multiple mechanisms, including DNA damage and induction of aging-related biologic pathways, such as telomerase activity, DNA hypermethylation, and stem cell exhaustion..$^{18}$ Hence, the age cutoffs assumed for the general population may not apply to patients with cancer. We postulate that this phenomenon may account for the high prevalence of GA impairments in our cohort aged $<65$ years.

In a prior study, Aleixo et $\mathrm{al}^{21}$ reported the prevalence of GA impairments among patients aged $<65$ years with early-stage breast cancer. Patients aged 50 to 64 years had a high prevalence of falls in the past 6 months (15\%), abnormal timed up-and-go test $>14$ seconds ( $12 \%$ ), and impaired IADLs (17\%). However, patients aged 50 to 64 years comprised $<10 \%$ of the study population and were derived from exercise intervention trials and compared with patients aged $\geq 65$ years derived from a prospective registry, thus raising a possibility of selection bias. In comparison, our entire cohort includes unselected patients from a cancer registry who underwent initial consultation at the gastrointestinal oncology clinic. However, we report similar findings, with comparable rates of GA impairments and frailty among patients in the 60-65 age group.

Limitations of our study include being a single-institution analysis limited to gastrointestinal malignancies, and therefore our findings may not be generalizable to other 


\begin{tabular}{|c|c|c|c|c|}
\hline Age, median (IQR) & $62(61-63)$ & $69(66-71)$ & $79(77-81)$ & $<.001$ \\
\hline Sex & & & & .16 \\
\hline Race & & & & .25 \\
\hline White/Caucasian & $91(71.1)$ & 147 (68.4) & $90(80.4)$ & \\
\hline Black/African American & $35(27.3)$ & $63(29.3)$ & $21(18.8)$ & \\
\hline Other & $2(1.6)$ & $4(1.9)$ & $0(0.0)$ & \\
\hline High school graduate & $29(22.7)$ & $59(27.4)$ & $29(25.9)$ & \\
\hline Associate/Bachelor's degree & $58(45.3)$ & $91(42.3)$ & $53(47.3)$ & \\
\hline Advanced degree & $12(9.4)$ & $19(8.8)$ & $13(11.6)$ & \\
\hline Unknown & $5(3.9)$ & $12(5.6)$ & $3(2.7)$ & \\
\hline Marital status & & & & .01 \\
\hline Single & $14(10.9)$ & $15(7.0)$ & $2(1.8)$ & \\
\hline Widowed/Divorced/Separated & $23(18.0)$ & $52(24.2)$ & $44(39.3)$ & \\
\hline Married & $86(67.2)$ & $138(64.2)$ & $62(55.4)$ & \\
\hline Unknown & $5(3.9)$ & $10(4.7)$ & $4(3.6)$ & \\
\hline Cancer stage & & & & .05 \\
\hline 1 & $9(7.0)$ & $15(7.0)$ & $10(8.9)$ & \\
\hline II & $17(13.3)$ & $36(16.7)$ & $33(29.5)$ & \\
\hline III & $39(30.5)$ & $58(27.0)$ & $25(22.3)$ & \\
\hline IV & $63(49.2)$ & 102 (47.4) & $42(37.5)$ & \\
\hline Unknown & $0(0.0)$ & $4(1.9)$ & $2(1.8)$ & \\
\hline Treatment status & & & & .27 \\
\hline Pretreatment & 101 (78.3) & 170 (79.1) & $96(85.7)$ & \\
\hline During treatment & $27(21.7)$ & $45(20.9)$ & $16(14.3)$ & \\
\hline
\end{tabular}

Abbreviation: IQR, interquartile range.

aOther includes anal cancer $(n=10)$, gastrointestinal stromal tumor $(n=14)$, appendicular cancer $(n=3)$, neuroendocrine carcinoma $(n=39)$, and gastrointestinal cancer not otherwise specified $(n=5)$.

settings/populations. The reason for limiting our study to gastrointestinal malignancies was to eliminate the possibility of selection bias as mentioned earlier. Nevertheless, we recognize that our cohort is still quite diverse and there may be substantial variation in the proportion of patients with GA impairment and frailty within individual cancer types and cancer stages. Almost half of our patients had stage IV disease, which may explain the high rate of GA impairments in our study. Notably, another study among patients with early-stage breast cancer reported similar 
Table 2. Overall and Domain-Specific Geriatric Impairment and Frailty

\begin{tabular}{|c|c|c|c|c|}
\hline Variable $^{a}$ & $\begin{array}{l}\text { 60-64 Years } \\
n(\%)\end{array}$ & $\begin{array}{l}\text { 65-74 Years } \\
n(\%)\end{array}$ & $\begin{array}{l}\geq 75 \text { Years } \\
\mathrm{n}(\%)\end{array}$ & $P$ Value $^{\mathrm{b}}$ \\
\hline Total, $\mathrm{N}^{\mathrm{c}}$ & 119 & 199 & 104 & \\
\hline $\begin{array}{l}\text { GA impairment count (0-13), } \\
\text { median (IQR) }\end{array}$ & $2(1-4)$ & $2(1-5)$ & $3(1-6)$ & .11 \\
\hline Frailty score, median (IQR) & $0.26(0.13-0.41)$ & $0.28(0.15-0.40)$ & $0.28(0.17-0.48)$ & .29 \\
\hline Robust & $46(38.2)$ & $70(34.0)$ & $29(26.6)$ & \\
\hline Prefrail & $33(26.8)$ & $62(30.1)$ & $36(33.0)$ & \\
\hline Frail & $43(35.0)$ & 74 (35.9) & $44(40.4)$ & \\
\hline IADL impairment $\geq 1$ & $60(47.2)$ & $111(52.1)$ & $66(58.9)$ & .20 \\
\hline$A D L$ impairment $\geq 1$ & $26(20.5)$ & $42(19.7)$ & $27(24.1)$ & .64 \\
\hline Cognitive impairment & $11(8.7)$ & $12(5.6)$ & $9(8.0)$ & .54 \\
\hline Anxiety & $23(18.1)$ & $44(20.7)$ & $17(15.2)$ & .50 \\
\hline Depression & $19(15.0)$ & $26(12.2)$ & $15(13.4)$ & .75 \\
\hline$\geq 3$ comorbidities & $48(39.0)$ & $112(56.3)$ & $59(55.7)$ & .006 \\
\hline$\geq 9$ medications & $23(19.0)$ & $50(25.3)$ & $29(27.1)$ & .29 \\
\hline
\end{tabular}

Abbreviations: $A D L$, activities of daily living; GA, geriatric assessment; IADL, instrumental activities of daily living; IQR, interquartile range; PS, performance status. aSeparate pairwise comparisons were conducted between age group 60-64 vs 65-74 years and between 60-64 vs $\geq 75$ years with overall similar findings, with the exception of greater comorbidity burden in the higher age group (results not shown).

${ }^{b}$ Represents unadjusted $P$ values. Additional sensitivity analyses were performed accounting for marital support and cancer stage (Mantel-Haenszel test using marital support and cancer stage as stratifying variables in case of categorical variables, Poisson and linear regression for GA impairment count and frailty score, respectively, controlling for marital support and cancer stage); however, the results remained unchanged (results not shown).

${ }^{\mathrm{C}}$ Total number of patients with available data to calculate the number of GA impairments. Patients were required to have nonmissing information on at least 11 domains $(85 \%)$ to have a valid result.

findings. ${ }^{21}$ All patients who underwent GA evaluation did so at the time of initial contact with the UAB health system. Consequently, not all patients who presented for an initial appointment were previously untreated, and approximately $20 \%$ had already undergone cancer therapy at another facility. Furthermore, by limiting our sample to patients completing GA at their initial visit, we may have excluded patients with severe illness requiring hospitalization for urgent treatment or hospice care, such as those with more aggressive malignancies, including hepatobiliary and pancreatic cancers. This may have potentially biased our findings. We did not have data on treatment-related toxicity, treatment discontinuation, or healthcare utilization, which need to be explored in future studies.

\section{Conclusions}

Our study adds to the growing body of evidence that chronologic age is an imperfect marker of presence of GA impairment and frailty. Furthermore, GA impairments are seen even among adults aged $<65$ years, and GA may aid in the clinical management of even younger populations than previously considered.

Submitted July 2, 2020; final revision received September 25, 2020, accepted for publication October 26, 2020. Published online June 11, 2021.

Previous presentation: This study was presented in abstract form at the 2020 ASCO Virtual Scientific Program; May 29-31, 2020. Abstract 12048.

Author contributions: Study concept and design: Giri, Williams. Data acquisition: Giri, Williams. Data analysis and interpretation: Giri, Williams. Statistical analysis: Giri, Williams. Supervision: Williams, Bhatia. Manuscript preparation: All authors. Critical revision: All authors.

Disclosures: Dr. Giri has disclosed receiving grant/research support from Carevive Systems and Pack Health LLC, and honoraria from Carevive Systems. Dr. Williams has disclosed serving as a consultant for Carevive Systems. The remaining authors have disclosed that they have not received any financial consideration from any person or organization to support the preparation, analysis, results, or discussion of this article.

Funding: This work was supported in part by the Walter B. Frommeyer Fellowship in Investigative Medicine at the University of Alabama at Birmingham and the NCl of the NIH (K08CA234225; G.R. Williams).

Disclaimer: The content is solely the responsibility of the authors and does not necessarily represent the official views of the $\mathrm{NIH}$.

Correspondence: Smith Giri, MD, MHS, Division of Hematology/Oncology, Institute of Cancer Outcomes and Survivorship, University of Alabama at Birmingham, 1600 7th Avenue South, Lowder 500, Birmingham, AL 35233. Email: smithgiri@uabmc.edu 


\section{References}

1. Smith BD, Smith GL, Hurria A, et al. Future of cancer incidence in the United States: burdens upon an aging, changing nation. J Clin Oncol 2009;27:2758-2765.

2. Soto-Perez-de-Celis E, Li D, Yuan Y, et al. Functional versus chronological age: geriatric assessments to guide decision making in older patients with cancer. Lancet Oncol 2018;19:e305-316.

3. Wildiers $H$, Heeren $P$, Puts $M$, et al. International Society of Geriatric Oncology consensus on geriatric assessment in older patients with cancer. J Clin Oncol 2014;32:2595-2603.

4. Guerard EJ, Deal AM, Chang Y, et al. Frailty Index developed from a cancer-specific geriatric assessment and the association with mortality among older adults with cancer. J Natl Compr Canc Netw 2017;15:894-902.

5. Hurria A, Togawa K, Mohile SG, et al. Predicting chemotherapy toxicity in older adults with cancer: a prospective multicenter study. J Clin Oncol $2011 ; 29: 3457-3465$

6. Lore Decoster LV, Kenis C, Prenen H, et al. Relevance of geriatric assessment in older patients with colorectal cancer. Clin Colorectal Cancer 2017;16:e221-229.

7. Caillet $P$, Canoui-Poitrine $F$, Vouriot J, et al. Comprehensive geriatric assessment in the decision-making process in elderly patients with cancer: ELCAPA study. J Clin Oncol 2011;29:3636-3642.

8. Mohile SG, Epstein RM, Hurria A, et al. Communication with older patients with cancer using geriatric assessment: a cluster-randomized clinical trial from the National Cancer Institute Community Oncology Research Program. JAMA Oncol 2020;6:196-204.

9. Reisinger KW, van Vugt JL, Tegels JJ, et al. Functional compromise reflected by sarcopenia, frailty, and nutritional depletion predicts adverse postoperative outcome after colorectal cancer surgery. Ann Surg 2015; 261:345-352.

10. Tegels JJ, de Maat MF, Hulsewé KW, et al. Value of geriatric frailty and nutritional status assessment in predicting postoperative mortality in gastric cancer surgery. J Gastrointest Surg 2014;18:439-446.

11. Wagner D, Büttner $S, \operatorname{Kim} Y$, et al. Clinical and morphometric parameters of frailty for prediction of mortality following hepatopancreaticobiliary surgery in the elderly. Br J Surg 2016;103:e83-92.

12. Aaldriks AA, van der Geest LG, Giltay EJ, et al. Frailty and malnutrition predictive of mortality risk in older patients with advanced colorectal cancer receiving chemotherapy. J Geriatr Oncol 2013;4:218-226.

13. Rostoft S. Integration of geriatric assessment in the care of patients with gastrointestinal malignancies. Visc Med 2017;33:275-280.

14. Siri R, Kristjansson AN, Marit $S$, et al. Comprehensive geriatric assessment can predict complications in elderly patients after elective surgery for colorectal cancer: a prospective observational cohort study. Crit Rev Oncol Hematol 2010;76:208-217.

15. Lee $\mathrm{YH}, \mathrm{Oh} \mathrm{HK}$, Kim DW, et al. Use of a comprehensive geriatric assessment to predict short-term postoperative outcome in elderly patients with colorectal cancer. Ann Coloproctol 2016;32:161-169.

16. Mohile SG, Dale W, Somerfield MR, et al. Practical assessment and management of vulnerabilities in older patients receiving chemotherapy: ASCO guideline for geriatric oncology. J Clin Oncol 2018;36:2326-2347.

17. Dotan E, Walter LC, Baumgartner J, et al. NCCN Clinical Practice Guidelines in Oncology: Older Adult Oncology. Version 1.2020. Accessed March 7, 2020. To view the most recent version, visit NCCN.org

18. Cupit-Link MC, Kirkland JL, Ness KK, et al. Biology of premature ageing in survivors of cancer. ESMO Open 2017;2:e000250.

19. Williams GR, Kenzik KM, Parman M, et al. Integrating geriatric assessment into routine gastrointestinal (GI) consultation: the Cancer and Aging Resilience Evaluation (CARE). J Geriatr Oncol 2020;11:270-273.
20. Mir N, MacLennan P, Al-Obaidi M, et al. Patient-reported cognitive complaints in older adults with gastrointestinal malignancies at diagnosis: results from the Cancer \& Aging Resilience Evaluation (CARE) study. J Geriatr Oncol 2020;11:982-988.

21. Aleixo GFP, Choi SK, Tan AJ, et al. Is "geriatric" assessment just for older patients? Oncologist 2020;25:355-358.

22. Fillenbaum GG, Smyer MA. The development, validity, and reliability of the OARS multidimensional functional assessment questionnaire. J Gerontol 1981;36:428-434.

23. Bauer J, Capra S, Ferguson M. Use of the scored Patient-Generated Subjective Global Assessment (PG-SGA) as a nutrition assessment tool in patients with cancer. Eur J Clin Nutr 2002;56:779-785.

24. Teno J, Kiel DP, Mor V. Multiple stumbles: a risk factor for falls in community-dwelling elderly. A prospective study. J Am Geriatr Soc 1990;38: 1321-1325.

25. Gabrielson DK, Scaffidi D, Leung E, et al. Use of an abridged scored Patient-Generated Subjective Global Assessment (abPG-SGA) as a nutritional screening tool for cancer patients in an outpatient setting. Nutr Cancer 2013;65:234-239.

26. Lees J, Chan A. Polypharmacy in elderly patients with cancer: clinical implications and management. Lancet Oncol 2011;12:1249-1257.

27. Klepin HD, Pitcher BN, Ballman KV, et al. Comorbidity, chemotherapy toxicity, and outcomes among older women receiving adjuvant chemotherapy for breast cancer on a clinical trial: CALGB 49907 and CALGB 361004 (Alliance). J Oncol Pract 2014;10:e285-292.

28. Moser A, Stuck AE, Silliman RA, et al. The eight-item modified Medical Outcomes Study Social Support Survey: psychometric evaluation showed excellent performance. J Clin Epidemiol 2012;65:1107-1116.

29. Pilkonis PA, Choi SW, Reise SP, et al. Item banks for measuring emotional distress from the Patient-Reported Outcomes Measurement Information System (PROMIS): depression, anxiety, and anger. Assessment 2011; 18:263-283.

30. Riley WT, Pilkonis P, Cella D. Application of the National Institutes of Health Patient-Reported Outcome Measurement Information System (PROMIS) to mental health research. J Ment Health Policy Econ 2011;14 201-208.

31. Saffer BY, Lanting SC, Koehle MS, et al. Assessing cognitive impairment using PROMIS applied cognition-abilities scales in a medical outpatient sample. Psychiatry Res 2015;226:169-172

32. Hays RD, Bjorner JB, Revicki DA, et al. Development of physical and mental health summary scores from the patient-reported outcomes measurement information system (PROMIS) global items. Qual Life Res 2009;18:873-880.

33. Kenis C, Decoster L, Van Puyvelde K, et al. Performance of two geriatric screening tools in older patients with cancer. J Clin Oncol 2014;32:19-26.

34. Rockwood K, Mitnitski A. Frailty in relation to the accumulation of deficits. J Gerontol A Biol Sci Med Sci 2007;62:722-727.

35. Searle SD, Mitnitski A, Gahbauer EA, et al. A standard procedure for creating a frailty index. BMC Geriatr 2008;8:24.

36. Cohen HJ, Smith D, Sun CL, et al. Frailty as determined by a comprehensive geriatric assessment-derived deficit-accumulation index in older patients with cancer who receive chemotherapy. Cancer 2016;122:3865-3872.

37. Mitnitski A, Song X, Skoog I, et al. Relative fitness and frailty of elderly men and women in developed countries and their relationship with mortality. J Am Geriatr Soc 2005;53:2184-2189.

38. Cuzick J. A Wilcoxon-type test for trend. Stat Med 1985;4:87-90

See JNCCN.org for supplemental online content. 
Supplemental online content for:

\section{Association Between Chronologic Age and Geriatric Assessment-Identified Impairments: Findings From the CARE Registry}

Smith Giri, MD, MHS; Mustafa Al-Obaidi, MBBS; Alice Weaver, MD; Kelly M. Kenzik, PhD;

Andrew McDonald, MD, MS; Deanna Clark, MBA, MPH; Crystal Young-Smith, CRNP;

Ravi Paluri, MD, MPH; Lakshmin Nandagopal, MD; Olumide Gbolahan, MD; Mackenzi Pergolotti, PhD;

Smita Bhatia, MD, MPH; and Grant R. Williams, MD

J Natl Compr Canc Netw 2021;19(8):922-927

eFigure 1: Study Cohort Selection

eTable 1: Overview of GA Measures by Domain

eTable 2: Baseline Demographic and Clinical Characteristics

eTable 3: Patterns of Comorbidity Burden Among Older Adults

eAppendix 1: Definition of GA Impairment

eAppendix 2: Construction of CARE Frailty Index 


\section{1 - Giri et al}

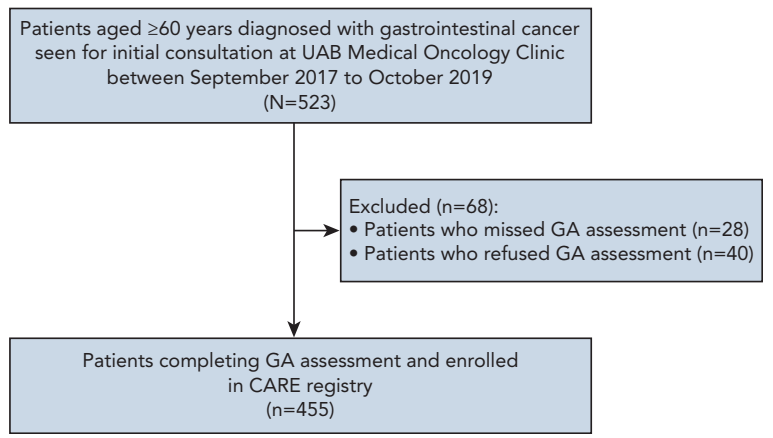

eFigure 1. Study cohort selection.

Abbreviations: CARE, Cancer and Aging Resilience Evaluation; GA, geriatric assessment; UAB, University of Alabama.

\section{eTable 1. Overview of GA Measures by Domain}

\begin{tabular}{|ll|}
\hline GA Domain & Patient-Reported Measures \\
\hline Function & $\begin{array}{l}\text { OARS IADLs } \\
\text { OARS ADLs } \\
\text { Patient-reported ECOG PS } \\
\text { Number of falls in last 6 mo }\end{array}$ \\
\hline Nutrition & Patient-generated subjective GA \\
\hline Cognition & PROMIS Cognitive Function \\
\hline Psychologic & $\begin{array}{l}\text { PROMIS Short Form v1.0 - Anxiety 4a } \\
\text { PROMIS Short Form v1.0 - Depression 4a }\end{array}$ \\
\hline Social support & $\begin{array}{l}\text { MOS-SSS } \\
\text { (Emotional/Informational Support subscales) }\end{array}$ \\
\hline Comorbidity & $\begin{array}{l}\text { Number of medications } \\
\text { OARS comorbidity assessment } \\
\text { Self-reported vision and hearing loss }\end{array}$ \\
\hline HRQoL & PROMIS Global-10 \\
\hline
\end{tabular}

Abbreviations: $A D L$, activities of daily living; $G A$, geriatric assessment; $H R Q \circ L$, health-related quality of life; IADL, instrumental activities of daily living; MOS-SSS, Medical Outcomes Study-Social Support Survey; OARS, Older Americans Resources and Services; PROMIS, Patient-Reported Outcomes Measurement Information System; PS, performance status. 


\section{eTable 2. Baseline Demographic and Clinical Characteristics}

\begin{tabular}{|c|c|c|c|}
\hline Variable & $\begin{array}{l}\text { Participants } \\
\text { n (\%) }\end{array}$ & $\begin{array}{l}\text { Nonparticipants } \\
\text { n (\%) }\end{array}$ & $P$ Value \\
\hline Total, N & 455 & 68 & \\
\hline Age, median (IQR) & $62(61-63)$ & $69(66-71)$ & .75 \\
\hline Age category & & & .94 \\
\hline $60-64$ y & $128(28.1)$ & $17(29.3)$ & \\
\hline $65-75$ y & $215(47.3)$ & $26(44.8)$ & \\
\hline$\geq 75$ y & $112(24.6)$ & $15(25.9)$ & \\
\hline Sex & & & .12 \\
\hline Male & $253(55.6)$ & $31(45.6)$ & \\
\hline Female & $202(44.4)$ & $37(54.4)$ & \\
\hline Race & & & $<.01$ \\
\hline White/Caucasian & $328(72.1 \%)$ & $48(70.6)$ & \\
\hline Black/African American & $119(26.2)$ & $13(19.1)$ & \\
\hline Other & $6(1.3)$ & $6(8.8)$ & \\
\hline Unknown & $2(0.4)$ & $1(1.5)$ & \\
\hline Cancer type & & & .02 \\
\hline Colorectal & $149(32.7)$ & $17(25.0)$ & \\
\hline Pancreatic & $108(23.7)$ & $23(33.8)$ & \\
\hline Hepatobiliary & $80(17.6)$ & $19(27.9)$ & \\
\hline Gastroesophageal & $47(10.3)$ & $5(7.4)$ & \\
\hline Other & $71(15.6)$ & $4(5.9)$ & \\
\hline Cancer stage & & & .26 \\
\hline I & $34(7.5)$ & $3(4.4)$ & \\
\hline II & $86(18.9)$ & $10(14.7)$ & \\
\hline III & $122(26.8)$ & $22(32.4)$ & \\
\hline IV & 207 (45.5) & $30(44.1)$ & \\
\hline Unknown & $6(1.3)$ & $4(2.5)$ & \\
\hline
\end{tabular}

Abbreviation: IQR, interquartile range. 


\section{eTable 3. Patterns of Comorbidity Burden Among Older Adults}

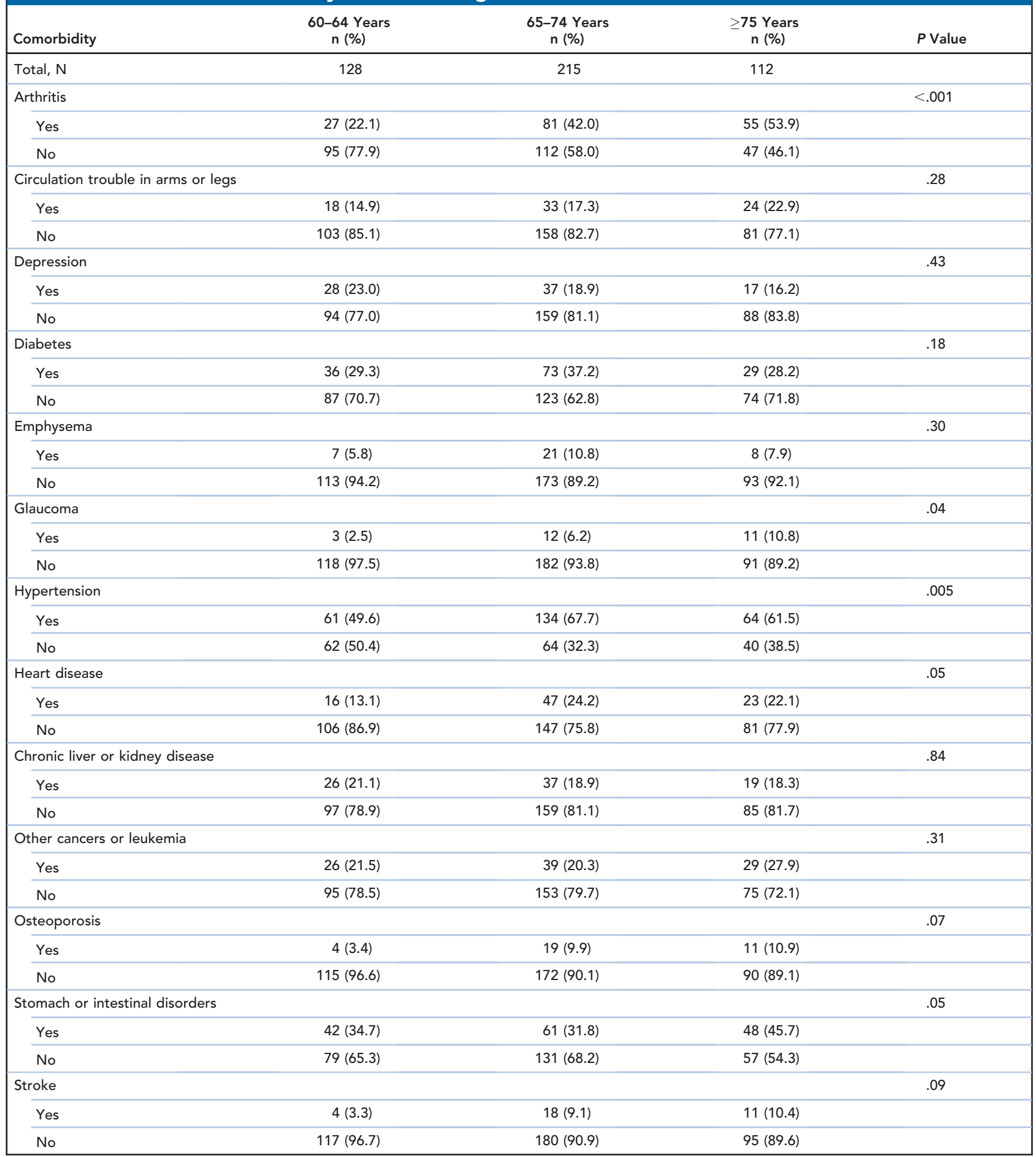




\section{eAppendix 1. Definition of GA Impairment}

GA impairment was defined as presence of $\geq 2$ of the following 13 domain-specific impairments. Using a cutoff of $\geq 2$ impairments was chosen to be consistent with prior publications. If a patient answered $\geq 11$ questions (85\%), we would calculate the number of GA impairments and categorize as presence/absence of GA impairment.

1. History of $\geq 1$ falls in the past 6 months.

2. Significant limitations in walking one block. Patients responding "limited a lot" to the question "Does your health limit you in walking one block?"

3. Presence of $\geq 2$ limitations in IADLs.

4. Presence of any impairment in ADLs.

5. Presence of significant weight loss. This is defined as $3 \%$ weight loss within 3 months or $6 \%$ weight loss within 6 months.

6. Patient-reported ECOG PS $\geq 3$ (ie, able to do little activity/pretty much bedridden).

7. Interference in social activity. Patient responding "most of the time or all of the time" to the question "During the past 4 weeks, how much of the time has your physical health or emotional problems interfered with your social activities (eg, visiting friends, relatives)?"

8. Multimorbidity defined as presence of $\geq 4$ comorbidities.

9. Significant impairment in any tangible support on the MOS-SSS. Patients responding as "none" or "a little" or "some of the time."

10. Anxiety defined as PROMIS Anxiety T-score $>60$.

11. Depression defined as PROMIS Depression T-score $>60$.

12. Cognitive impairment defined as PROMIS Cognitive Function T-score $<40$.

13. Polypharmacy defined as taking $\geq 9$ daily medications.

Abbreviations: ADL, activities of daily living; GA, geriatric assessment; IADL, instrumental activities of daily living; MOS-SSS, Medical Outcomes Study-Social Support Survey; PROMIS, Patient-Reported Outcomes Measurement Information System; PS, performance status. 


\section{eAppendix 2. Construction of CARE Frailty Index}

We constructed a frailty index (the CARE Frailty Index) using the principle of deficit accumulation approach originally described by Rockwood and Mitnitski ${ }^{1}$ and following the standard procedures outlined by Searle et al. ${ }^{2}$ Similar methods have been used by Guerard et $\mathrm{al}^{3}$ and Cohen et al ${ }^{4}$ to construct frailty indices that have been shown to be predictive of chemotherapy toxicity ${ }^{4}$ and all-cause mortality ${ }^{3}$ among older adults with cancer. We selected 44 GA variables from the CARE questionnaire, each of which captured a health deficit, and recoded responses using the convention that 0 indicated the absence of the deficit and 1 indicated the presence of deficit; for variables that included a single intermediate response (eg, "sometimes" or "maybe"), we used an additional value of 0.5 . We then combined these individual scores into an aggregate frailty score reflecting the overall proportion of deficits (range, $0-1)$, where $0=$ no deficit present and $1=$ all 44 deficits present. We then categorized patients as robust $(<0.2)$, prefrail $(0.2-0.35)$, and frail $(>0.35)$, as previously described. ${ }^{2}$ In case of missing response data, we required that responses to at least 30 items be present to construct a valid frailty index. An index constructed with at least 30 variables has been previously shown to be sufficiently accurate for predicting adverse outcomes among older adults. ${ }^{5}$

The 44 variables used for construction of CARE Frailty Index are as follows:

1. Falls $\geq 1: 1$ point

2. Walk one block = limited a lot: 1 point

3. IADL mobility (unable to/with some help): 1 point

4. IADL shopping (unable to/with some help): 1 point

5. IADL meal prepare (unable to/with some help): 1 point

6. IADL housework (unable to/with some help): 1 point

7. IADL medication (unable to/with some help): 1 point

8. IADL money (unable to/with some help): 1 point

9. $A D L$ get in and out of bed (unable to/with some help): 1 point

10. ADL dress (unable to/with some help): 1 point

11. ADL bath (unable to/with some help): 1 point

12. Global health: good $=0.5$ point, fair/poor $=1$ point

13. Global quality of life: good $=0.5$ point, fair/poor $=1$ point

14. Global physical health: good $=0.5$ point, fair/poor $=1$ point

15. Global mental health: good $=0.5$ point, fair/poor $=1$ point

16. Global satisfaction with social activities and relationship: good $=0.5$ point, fair/poor $=1$ point

17. Global everyday activities: moderately $=0.5$ point, a little/not at all $=1$ point

18. Global anxious/depression: sometimes $=0.5$ point, often/always $=1$ point

19. Global fatigue: moderate $=0.5$ point, severe/very severe $=1$ point

20. Global pain: pain level $4-6=0.5$ point, pain level 7-10 $=1$ point

21. Global social activities and roles: good $=0.5$ point, fair $/$ poor $=1$ point

22. Weight loss: 3 - or 6-month weight loss $\geq 5 \%$ : 1 point

23. Food intake less than usual: 1 point

24. Activities and function (self-rated activity) $\geq 2$ (in bed or chair less than half the day/able to do little activity/ pretty much bedridden): 1 point

25. Anxiety PROMIS T-score $>60: 1$ point

26. Depression PROMIS T-score $>60: 1$ point

27. Impaired Cognition: PROMIS T-score $<40$ : 1 point

28. Number of daily medications $\geq 9$ : 1 point

29. Social activity interference: some of the time $=0.5$ point, most/all of the time $=1$ point

\section{Comorbidities}

30. Eyesight fair/poor/totally blind: 1 point

31. Hearing fair/poor/totally deaf: 1 point

32. Other cancers or leukemia: 1 point

33. Arthritis or rheumatism: 1 point

34. Glaucoma: 1 point

35. Emphysema or chronic bronchitis: 1 point

36. High blood pressure: 1 point 


\section{eAppendix 2. Construction of CARE Frailty Index (cont.)}

37. Heart disease: 1 point

38. Circulation trouble in arms or legs: 1 point

39. Diabetes: 1 point

40. Stomach or intestinal disorders: 1 point

41. Osteoporosis: 1 point

42. Chronic liver or kidney disease: 1 point

43. Stroke: 1 point

44. Depression: 1 point

Abbreviations: ADL, activities of daily living; CARE, Cancer and Aging Resilience Evaluation; GA, geriatric assessment; IADL, instrumental activities of daily living; PROMIS, Patient-Reported Outcomes Measurement Information System.

\section{References}

1. Rockwood K, Mitnitski A. Frailty in relation to the accumulation of deficits. J Gerontol A Biol Sci Med Sci 2007;62:722-727.

2. Searle SD, Mitnitski A, Gahbauer EA, et al. A standard procedure for creating a frailty index. BMC Geriatr 2008;8:24.

3. Cohen HJ, Smith D, Sun CL, et al. Frailty as determined by a comprehensive geriatric assessment-derived deficit-accumulation index in older patients with cancer who receive chemotherapy. Cancer 2016;122:3865-3872.

4. Mitnitski A, Song X, Skoog I, et al. Relative fitness and frailty of elderly men and women in developed countries and their relationship with mortality. J Am Geriatr Soc 2005;53:2184-2189.

5. Cuzick J. A Wilcoxon-type test for trend. Stat Med 1985;4:87-90. 